International Mathematical Forum, 1, 2006, no. 35, 1709-1727

\title{
Some generalisations of Birnbaum-Saunders and sinh-normal distributions
}

\author{
José A. Díaz-García \\ Universidad Autónoma Agraria Antonio Narro \\ Department of Statistics and Computation \\ 25315 Buenavista, Saltillo \\ Coahuila, México \\ jadiaz@uaaan.mx \\ José Ramón Domínguez-Molina \\ Centro de Investigación en Matemáticas, A.C. \\ Gerencia de Consultoría Estadística \\ 36240, Jalisco S/N, Mineral de Valenciana \\ Guanajuato, Guanajuato, México \\ jrdguez@cimat.mx
}

\begin{abstract}
This work proposes the univariate and multivariate generalisations of the following distributions: the Birnbaum-Saunders, the three parameter Birnbaum-Saunders and the sinh-normal of spherical type. Similarly, when the stochastic representation of a spherical random vector is assumed, we propose alternative definitions for the above-mentioned distributions including the log-elliptical distribution. We emphasize that all the distributions here derived belong to the family of the spherical distributions.
\end{abstract}

Mathematics Subject Classification: 62E15, 62N05

Keywords: Birnbaum-Saunders distribution, life distributions, distributions, sinh-normal, stochastic representation, multivariate log-elliptical distribution

\section{Introduction}

Recently, the family of elliptical contour distributions have been a vertiginous development in many areas of the statistics; the main topic to research goes 
around the extension of the models and techniques of the statistical literature when the distributions are elliptical. The books of [13], [11] and [12] give an excellent summary of that literature development. Topics in the context of distribution theory and sensibility analysis have been treated by [6] and [8], among many others. Specifically, in statistical theory of reliability [10] generalise the Birnbaum-Saunders distribution under the spherical case. More recently, [5] propose a family of distributions based on spherical distributions for a dependent sample of life data. Another life distribution is the log-normal distribution, see [19], this was generalised by [11, p. 55] to the multivariate elliptical case.

In the present work: the multivariate generalisation of the BirnbaumSaunders distribution, under an elliptical distribution, is given in Section 3. In Section 4 we propose the univariate and multivariate generalisations of the three parameter Birnbaum-Saunders distribution (see [20]). In Section 5, the univariate and multivariate sinh-spherical distributions are derived (they are known as the generalised log-Birnbaum-Saunders distributions). Section 6 extends the results of Section 5 when the three parameter Birnbaum-Saunders distribution is considered. In Section 7 we propose alternative definitions for the Birnbaum-Saunders, the log-elliptical and the sinh-spherical multivariate distributions, in particular, when the stochastic representation of a random vector with spherical distribution is considered and noting that such representation coincides with some algebraic factorizations of that vector; we emphasized that the new family of distributions continue belonging the family of spherical distributions; the section ends with the extension of the new families to the elliptical case. Finally, in order to check the parameter estimations of the new families, we took some sets of data given in the literature for the independent case, and we simulated a set of life data for the dependent case; then the maximum likelihood estimators of the parameters under particular distributions are obtained, see Section 8.

\section{Preliminary considerations}

In this section some preliminary results are given as a necessary background for the development of the paper.

Suppose that $Z \sim \mathcal{N}(0,1)$, and let $\alpha>0$ and $\beta>0$. The random variable

$$
S=\beta\left[\frac{\alpha}{2} Z+\sqrt{\left(\frac{\alpha}{2} Z\right)^{2}+1}\right]^{2}
$$

has a distribution known as the Birnbaum-Saunders distribution, which is 
denoted by $S \sim \mathcal{B S}(\alpha, \beta)$. More over, its density function is given by

$$
f_{S}(s)=\frac{s^{-3 / 2}(s+\beta)}{2 \alpha(2 \pi \beta)^{1 / 2}} \exp \left\{-\frac{1}{2 \alpha^{2}}\left(\frac{s}{\beta}+\frac{\beta}{s}-2\right)\right\}, \quad s>0
$$

where $\alpha$ is the shape parameter and $\beta$ is the scale parameter and the median of the distribution, see [3].

A generalisation of the Birnbaum-Saunders distribution was proposed by [20]; in that case, the inverse transformation of (1) is defined by

$$
Z=\frac{1}{\alpha}\left[\left(\frac{v}{\beta}\right)^{\lambda}-\left(\frac{\beta}{v}\right)^{\lambda}\right], \lambda>0
$$

Note that when $\lambda=0.5$ we get as a particular case the Birnbaum-Saunders distribution.

The generalisation of the Birnbaum-Saunders distribution is termed the three parameter Birnbaum-Saunders distribution and its density is given by

$$
f_{V}(v)=\frac{\lambda}{\alpha v(2 \pi)^{1 / 2}}\left[\left(\frac{v}{\beta}\right)^{\lambda}+\left(\frac{\beta}{v}\right)^{\lambda}\right] \exp \left\{-\frac{1}{2 \alpha^{2}}\left[\left(\frac{v}{\beta}\right)^{2 \lambda}+\left(\frac{\beta}{v}\right)^{2 \lambda}-2\right]\right\}
$$

$v>0$; and we shall denoted by $V \sim \mathcal{G B}-\mathcal{S}(\alpha, \beta, \lambda)$.

A very close distribution to the Birnbaum-Saunders is the sinh-normal distribution, and it plays an important role in the log-linear models for life data with Birnbaum-Saunders distribution. The density of a sinh-normal distribution is given by

$$
f_{Y}(y)=\frac{2}{\alpha \sigma \sqrt{2 \pi}} \cosh \left(\frac{y-\gamma}{\sigma}\right) \exp \left\{-\frac{2}{\alpha^{2}} \sinh ^{2}\left(\frac{y-\gamma}{\sigma}\right)\right\}, \quad y \in \Re,
$$

and it is denoted by $Y \sim \mathcal{S N}(\alpha, \gamma, \sigma)$, where $\gamma \in \Re$ is a location parameter, $\sigma>0$ is the scale parameter and $\alpha>0$ is the shape parameter. This distribution and some properties are studied in [22].

By other side, we say that a $p$-dimensional random vector $\mathbf{Y}=\left(Y_{1}, \ldots, Y_{p}\right)^{\prime}$ has an elliptical distribution with position parameter $\boldsymbol{\mu}: p \times 1$ and scale parameter $\Sigma: p \times p, \Sigma>0$, if its density function is given by

$$
f_{\mathbf{Y}}(\mathbf{y})=c|\boldsymbol{\Sigma}|^{-1 / 2} h\left\{(\mathbf{y}-\boldsymbol{\mu})^{\prime} \boldsymbol{\Sigma}^{-1}(\mathbf{y}-\boldsymbol{\mu})\right\}, \quad \mathbf{y} \in \Re^{p},
$$

where the function $h: \Re \rightarrow[0, \infty)$ is termed the function generator and it is such that $\int_{0}^{\infty} u^{p-1} h\left(u^{2}\right) d u<\infty$ with $c$ a proportionality constant, so that $f_{\mathbf{Y}}(\mathbf{y})$ is a density. It shall be denoted by $\mathbf{Y} \sim \mathcal{E} l_{p}(\boldsymbol{\mu}, \boldsymbol{\Sigma} ; h)$. When the vector $\mathbf{Y}$ has finite moments, we have that $E(\boldsymbol{Y})=\boldsymbol{\mu}$ and $\operatorname{Var}(\boldsymbol{Y})=c_{h} \boldsymbol{\Sigma}$, where $c_{h}$ is a positive constant, see for example [12] or [11]. In the particular case when 
$\boldsymbol{\mu}=\mathbf{0}$ and $\boldsymbol{\Sigma}=\boldsymbol{I}_{p}$, we get the family of spherical distributions, they shall denote by $\mathbf{Y} \sim \mathcal{E}_{p}(\mathbf{0}, \mathbf{I} ; h)$. These distributions include as particular cases the following distributions: the Normal, the $t$-Student, the Pearson type VII, the Logistic, the Bessel y Kotz, among many others.

Let $\Re_{+}^{p}$ be the positive part of $\Re^{p}$ and $\ln (\mathbf{W})=\left(\ln \left(w_{1}\right), \ldots, \ln \left(w_{p}\right)\right)^{\prime}$. Suppose that $\mathbf{w} \in \Re_{+}^{p}$ be a random vector such that $\log (\mathbf{W}) \sim \mathcal{E} l_{p}(\boldsymbol{\mu}, \boldsymbol{\Sigma} ; h)$; then we say that the vector $\mathbf{W}$ has a multivariate log-elliptical distribution Type $I$ if its density function is given by

$$
f_{\mathbf{W}}(\mathbf{W})=c|\mathbf{\Sigma}|^{-1 / 2}\left(\prod_{i=1}^{p} w_{i}^{-1}\right) h\left\{(\log (\mathbf{w})-\boldsymbol{\mu})^{\prime} \boldsymbol{\Sigma}^{-1}(\log (\mathbf{w})-\boldsymbol{\mu})\right\}, \quad \mathbf{w} \in \Re_{+}^{p},
$$

and it shall be denoted by $\mathbf{W} \sim \mathcal{L} \mathcal{E} l_{p}^{I}(\boldsymbol{\mu}, \boldsymbol{\Sigma} ; h)$.

Now let us consider the transformation (1) when the normality is substituted by a spherical law, this is, define

$$
T=\beta\left[\frac{\alpha}{2} U+\sqrt{\left(\frac{\alpha}{2} U\right)^{2}+1}\right]^{2}
$$

by assuming that $U \sim \mathcal{E}_{1}(0,1 ; h)$, then $T$ the following density function

$$
f_{T}(t)=\frac{c t^{-3 / 2}(t+\beta)}{2 \alpha \beta^{1 / 2}} h\left\{\frac{1}{\alpha^{2}}\left(\frac{t}{\beta}+\frac{\beta}{t}-2\right)\right\}, \quad t>0 .
$$

This is termed the generalised Birnbaum-Saunders distribution and it shall be denoted by $T \sim \mathcal{G B S}(\alpha, \beta ; h),[10]$.

\section{Birnbaum-Saunders distribution}

In this section we give some generalisations of the Birnbaum-Saunders distribution to the multivariate case.

Theorem 3.1 Suppose that $\mathbf{U} \sim \mathcal{E}_{n}(\mathbf{0}, \mathbf{I} ; h)$ and define the transformation

$$
T_{i}=\beta_{i}\left(\frac{1}{2} \alpha_{i} U_{i}+\sqrt{\left(\frac{1}{2} \alpha_{i} U_{i}\right)^{2}+1}\right)^{2}, \quad \alpha_{i}>0, \quad \beta_{i}>0, \quad i=1, \ldots, n ;
$$

the distribution of the vector $\mathbf{T}=\left(T_{1}, \ldots, T_{n}\right)^{\prime}$ is termed the multivariate generalised Birnbaum-Saunders distribution type I, and its density function is given by

$$
f_{\mathbf{T}}(\mathbf{t})=\frac{c}{2^{n}}\left(\prod_{i=1}^{n} \frac{t_{i}^{-3 / 2}\left(t_{i}+\beta_{i}\right)}{\alpha_{i} \sqrt{\beta_{i}}}\right) h\left\{\sum_{i=1}^{n} \frac{1}{\alpha_{i}^{2}}\left(\frac{t_{i}}{\beta_{i}}+\frac{\beta_{i}}{t_{i}}-2\right)\right\}, \mathbf{t} \in \Re_{+}^{n},
$$


and it is denoted by $\mathbf{T} \sim \mathcal{G B S}_{n}^{I}(\boldsymbol{\alpha}, \boldsymbol{\beta} ; h)$, with $\boldsymbol{\alpha}=\left(\alpha_{1}, \ldots, \alpha_{n}\right)^{\prime}$ and $\boldsymbol{\beta}=$ $\left(\beta_{1}, \ldots, \beta_{n}\right)^{\prime}$.

Proof: Given that $\mathbf{U} \sim \mathcal{E}_{n}(0, I, ; h)$, the density of $\mathbf{U}$ is given by

$$
f_{\mathbf{U}}(\mathbf{u})=c h\left(\|\mathbf{u}\|^{2}\right) .
$$

The proof follows easily observing that:

$$
\begin{aligned}
u_{i} & =\frac{1}{\alpha_{i}}\left(\sqrt{\frac{t_{i}}{\beta_{i}}}-\sqrt{\frac{\beta_{i}}{t_{i}}}\right) \quad i=1, \ldots, n, \\
\left|\frac{\partial u_{i}}{\partial t_{i}}\right| & =\frac{1}{2^{n}} \prod_{i=1}^{n} \frac{t_{i}^{-3 / 2}\left(t_{i}+\beta_{i}\right)}{\alpha_{i} \sqrt{\beta_{i}}} \\
\|u\|^{2} & =\sum_{i=1}^{n} \frac{1}{\alpha_{i}^{2}}\left(\frac{t_{i}}{\beta_{i}}+\frac{\beta_{i}}{t_{i}}-2\right) .
\end{aligned}
$$

Remark 3.2 An interesting particular case of the distribution (4) appears when $\alpha_{1}=\cdots=\alpha_{n}=\alpha$ and $\beta_{1}=\cdots=\beta_{n}=\beta$, which implies that $\boldsymbol{\alpha}=$ $\alpha \mathbf{1} \boldsymbol{\beta}=\beta \mathbf{1}$ with $\mathbf{1}=(1, \ldots, 1)^{\prime}$; because if the random vector $T$ denotes $a$ random sample of a univariate population, then the density of (4) defines a likelihood function when there is stochastic dependency in the random sample $T$. This idea and other particular cases and the estimation of the multivariate generalised Birnbaum-Saunders distribution type I can be found in [5].

\section{Three parameter Birnbaum-Saunders distri- bution}

Now we propose the univariate and multivariate extensions of the three parameter Birnbaum-Saunders distribution under a spherical model.

Theorem 4.1 Suppose that $U \sim \mathcal{E}(0,1 ; h)$ and consider the transformation

$$
U=\frac{1}{\alpha}\left[\left(\frac{v}{\beta}\right)^{\lambda}-\left(\frac{\beta}{v}\right)^{\lambda}\right], \lambda>0, \quad \beta>0, \quad \alpha>0
$$

the distribution of the random variable $V$ is termed generalised three parameter Birnbaum-Saunders distribution, and its density function is the following expression:

$$
f_{V}(v)=\frac{c \lambda}{\alpha v}\left[\left(\frac{v}{\beta}\right)^{\lambda}+\left(\frac{\beta}{v}\right)^{\lambda}\right] h\left\{\frac{1}{\alpha^{2}}\left[\left(\frac{v}{\beta}\right)^{2 \lambda}+\left(\frac{\beta}{v}\right)^{2 \lambda}-2\right]\right\}, v>0
$$

this shall be denoted by $V \sim \mathcal{G G B}-\mathcal{S}(\alpha, \beta, \lambda ; h)$. 
Proof: The demonstration follows by noting that

$$
d u=\frac{\lambda}{\alpha v}\left(\frac{v^{2 \lambda}+\beta^{2 \lambda}}{(\beta v)^{\lambda}}\right)
$$

Also, observe that this distribution keeps the reciprocal property: $V^{-1} \sim$ $\mathcal{G G B}-\mathcal{S}(\alpha, 1 / \beta, \lambda ; h)$

The generalisation of that distribution to the multivariate case is immediate:

Theorem 4.2 Suppose that $\mathbf{U} \sim \mathcal{E}_{n}(\mathbf{0}, \mathbf{I} ; h)$, and consider the transformation (5), for $u_{i}$, and $\alpha_{i}>0, \beta_{i}>0$ and $\lambda_{i}>0, i=1, \ldots, n$; the density function of the random vector $\mathbf{V}=\left(v_{1}, \ldots v_{n}\right)^{\prime}$ is given by

$f_{\mathbf{V}}(v)=c \prod_{i=1}^{n} \frac{\lambda_{i}}{\alpha_{i} v_{i}}\left[\left(\frac{v_{i}}{\beta_{i}}\right)^{\lambda_{i}}+\left(\frac{\beta_{i}}{v_{i}}\right)^{\lambda_{i}}\right] h\left\{\sum_{i=1}^{n} \frac{1}{\alpha_{i}^{2}}\left[\left(\frac{v_{i}}{\beta_{i}}\right)^{2 \lambda_{i}}+\left(\frac{\beta_{i}}{v_{i}}\right)^{2 \lambda_{i}}-2\right]\right\}$,

$\mathbf{v} \in \Re_{+}^{n}$. Writing $\boldsymbol{\alpha}=\left(\alpha_{1}, \ldots, \alpha_{n}\right)^{\prime}, \boldsymbol{\beta}=\left(\beta_{1}, \ldots, \beta_{n}\right)^{\prime}$ and $\boldsymbol{\lambda}=\left(\lambda_{1}, \ldots, \lambda_{n}\right)^{\prime}$; we shall call it the multivariate generalised three parameter Birnbaum-Saunders distribution type I and it shall denote by $\mathbf{V} \sim \mathcal{G G B}-\mathcal{S}_{n}^{I}(\boldsymbol{\alpha}, \boldsymbol{\beta}, \boldsymbol{\lambda} ; h)$.

Remark 4.3 An analogous observation to Remark 3.2 can be established for the multivariate generalised three parameter Birnbaum-Saunders distribution type I, seen its role as a likelihood function in the case of a dependent sample.

\section{Sinh-spherical distribution}

Let $G_{U}(u)$ be denotes the distribution function of the random variable $U \sim$ $\mathcal{E}(0,1 ; h)$. By analogy to the case of a sinh-normal distribution( see [22]), the distribution function $F_{Y}(y)$, of the random variable $Y$ with sinh-spherical distribution, is given by

$$
F_{Y}(y)=G_{U}\left(\frac{2}{\alpha} \sinh \left(\frac{y-\gamma}{\sigma}\right)\right)
$$

where $\alpha>0, \sigma>0$ and $\gamma \in \Re$. So, deriving with respect to $y$ we obtain

$$
f_{Y}(y)=\frac{2 c}{\alpha \sigma} \cosh \left(\frac{y-\gamma}{\sigma}\right) h\left\{\frac{4}{\alpha^{2}} \sinh ^{2}\left(\frac{y-\gamma}{\sigma}\right)\right\}, \quad y \in \Re
$$

this distribution shall termed the generalised sinh-spherical distribution and it shall be denoted by $Y \sim \mathcal{S E}(\alpha, \gamma, \sigma)$.

As in the case of the sinh-normal distribution, the sinh-spherical distribution is related with the generalised Birnbaum-Saunders distribution. 
Theorem 5.1 Suppose that $T \sim \mathcal{G B S}(\alpha, \beta ; h)$, then $Y=\frac{\sigma}{2} \ln T$ has a sinhspherical distribution with shape, location and scale parameters given by $\alpha$, $\gamma=\frac{\sigma}{2} \ln \beta$ and $\sigma$, respectively.

Next, we find the first version of a multivariate generalised sinh-spherical distribution.

Theorem 5.2 Consider $\mathbf{T} \sim \mathcal{G B S}_{n}(\boldsymbol{\alpha}, \boldsymbol{\beta} ; h)$ and let $\mathbf{Y}=\frac{1}{2} \boldsymbol{\Sigma} \ln \mathbf{T}$, where $\Sigma: n \times n$ is a non-random positive definite matrix. We say that $Y$ has a multivariate sinh-spherical distribution type I of parameters $\boldsymbol{\alpha} \in \Re_{+}^{n}, \boldsymbol{\gamma}=$ $\frac{1}{2} \boldsymbol{\Sigma} \ln \boldsymbol{\beta} \in \Re^{n}$ and $\boldsymbol{\Sigma}>0$, and its density function is given by

$$
\begin{aligned}
f_{\mathbf{Y}}(\mathbf{y})=2^{n} c \prod_{i=1}^{n}\left[\frac{\mathbf{e}_{i}^{\prime} \boldsymbol{\Sigma}^{-1} \mathbf{e}_{i}}{\alpha_{i}} \cosh \left(\mathbf{e}_{i}^{\prime} \boldsymbol{\Sigma}^{-1}(\mathbf{y}-\gamma)\right)\right] \\
h\left\{\sum_{i=1}^{n} \frac{4}{\alpha_{i}^{2}} \sinh ^{2}\left(\mathbf{e}_{i}^{\prime} \boldsymbol{\Sigma}^{-1}(\mathbf{y}-\gamma)\right)\right\},
\end{aligned}
$$

with $\mathbf{y} \in \Re^{n}$, and $\mathbf{e}_{i}$ is the $i$-th vector of the canonical base in $\Re^{n}$. This fact shall be written as $\mathbf{Y} \sim \mathcal{S E}_{n}^{I}(\boldsymbol{\alpha}, \boldsymbol{\gamma}, \boldsymbol{\Sigma} ; h)$.

Proof: Define $\boldsymbol{\Sigma}$ such that

$$
\Sigma^{-1}=\left(\sigma_{i j}^{*}\right)=\left(\begin{array}{c}
\Sigma_{1}^{*^{\prime}} \\
\vdots \\
\Sigma_{n}^{*^{\prime}}
\end{array}\right), \quad \Sigma_{i}^{*} \in \Re^{n}, \quad i=1, \ldots, n
$$

and noting that $\sigma_{i j}^{*}=\mathbf{e}_{i} \boldsymbol{\Sigma}^{-1} \mathbf{e}_{j}^{\prime}$ and $\boldsymbol{\Sigma}_{i}^{*}=\boldsymbol{\Sigma}^{-1} \mathbf{e}_{i}$. Thus, if $\mathbf{T}=\exp \left\{2 \boldsymbol{\Sigma}^{-1} \mathbf{Y}\right\}$,

$$
(d \mathbf{T})=2^{n} \prod_{i=1}^{p} \mathbf{e}_{i} \boldsymbol{\Sigma}^{-1} \mathbf{e}_{i}^{\prime} \exp \left\{2 \mathbf{e}_{i}^{\prime} \boldsymbol{\Sigma}^{-1} \mathbf{Y}\right\}(d \mathbf{Y}),
$$

where $(d \mathbf{T})=\bigwedge_{i=1}^{p} d t_{i}$ denotes the exterior product of the differential elements of the vector $d \mathbf{T}$, see [?, pp. 52-53]. The proof follows by making the change of variable $\boldsymbol{\gamma}=\frac{1}{2} \boldsymbol{\Sigma} \ln \boldsymbol{\beta}$ in the expression (4).

Interesting particular cases of the distribution (8) are obtained when:

1. $\boldsymbol{\Sigma}=\operatorname{diag}\left(\sigma_{1}, \ldots, \sigma_{n}\right)$, in this occasion

(a) $\boldsymbol{\Sigma}^{-1}=\operatorname{diag}\left(\sigma_{1}^{-1}, \ldots, \sigma_{n}^{-1}\right)$

(b) $\mathbf{e}_{i}^{\prime} \boldsymbol{\Sigma}^{-1} \mathbf{e}_{i}=\sigma_{i}^{-1}$

(c) $\mathbf{e}_{i}^{\prime} \boldsymbol{\Sigma}^{-1}(\mathbf{y}-\gamma)=\frac{y_{i}-\gamma_{i}}{\sigma_{i}}$ 
then

$$
f_{\mathbf{Y}}(\mathbf{y})=c 2^{n}\left(\prod_{i=1}^{n} \frac{\cosh \left(\frac{y_{i}-\gamma_{i}}{\sigma_{i}}\right)}{\sigma_{i} \alpha_{i}}\right) h\left\{4 \sum_{i=1}^{n} \frac{1}{\alpha_{i}^{2}} \sinh ^{2}\left(\frac{y_{i}-\gamma_{i}}{\sigma_{i}}\right)\right\}
$$

2. $\boldsymbol{\Sigma}=\sigma \mathbf{I}_{n}, \gamma_{i}=\gamma$ y $\alpha_{i}=\alpha$ for every $i=1, \ldots, n$, then

(a) $\boldsymbol{\Sigma}^{-1}=\sigma^{-1} \mathbf{I}_{n}$

(b) $\mathbf{e}_{i}^{\prime} \boldsymbol{\Sigma}^{-1} \mathbf{e}_{i}=\sigma^{-1}$

(c) $\mathbf{e}_{i}^{\prime} \boldsymbol{\Sigma}^{-1}(\mathbf{y}-\gamma)=\frac{y_{i}-\gamma}{\sigma}$

thus

$$
f_{\mathbf{Y}}(\mathbf{y})=\frac{2^{n} c}{\sigma^{n} \alpha^{n}}\left(\prod_{i=1}^{n} \cosh \left(\frac{y_{i}-\gamma}{\sigma}\right)\right) h\left\{\frac{4}{\alpha^{2}} \sum_{i=1}^{n} \sinh ^{2}\left(\frac{y_{i}-\gamma}{\sigma}\right)\right\} .
$$

\section{Sinh-spherical distribution under three pa- rameter birnbaum-Saunders distyribution}

Now we propose the univariate and multivariate versions of the sinh-spherical distribution under the three parameter Birnbaum-Saunders distribution.

Theorem 6.1 If $V \sim \mathcal{G G B}-\mathcal{S}(\alpha, \beta, \lambda ; h)$, then $Y=\frac{\sigma}{2} \ln V$ has a generalised sinh-spherical distribution with shape, location and scale parameters given by $\alpha, \gamma=\frac{\sigma}{2} \ln \beta$ and $\sigma$, respectively, where the parameter $\lambda>0$. Its density function is the following:

$$
f_{Y}(y)=\frac{4 c \lambda}{\alpha \sigma} \cosh \left[2 \lambda\left(\frac{y-\gamma}{\sigma}\right)\right] h\left\{\frac{4}{\alpha^{2}} \sinh ^{2}\left[2 \lambda\left(\frac{y-\gamma}{\sigma}\right)\right]\right\}, y \in \Re .
$$

This shall be denoted by $Y \sim \mathcal{G S E}(\alpha, \gamma, \sigma, \lambda ; h)$.

Proof. This is straightforward from Theorem 4.1 and noting that

$$
d v=\frac{2}{\sigma} \exp (2 y / \sigma) d y
$$

Next we write the multivariate extension: 
Theorem 6.2 Consider $\mathbf{V} \sim \mathcal{G B}-\mathcal{S}_{n}^{I}(\boldsymbol{\alpha}, \boldsymbol{\beta}, \boldsymbol{\lambda} ; h)$ and let $\mathbf{Y}=\frac{1}{2} \boldsymbol{\Sigma} \ln \mathbf{V}$, where $\boldsymbol{\Sigma}: n \times n$ is a non-random positive definite matrix, then, we say $Y$ has a multivariate generalised sinh-spherical distribution type I of parameters $\boldsymbol{\alpha}, \boldsymbol{\lambda} \in \Re_{+}^{n}, \boldsymbol{\gamma}=\frac{1}{2} \boldsymbol{\Sigma} \ln \boldsymbol{\beta} \in \Re^{n}$ and $\boldsymbol{\Sigma}>0$; and its density function is given by

$$
\begin{aligned}
f_{\mathbf{Y}}(\mathbf{y})=2^{2 n} c \prod_{i=1}^{n}\left[\frac{\mathbf{e}_{i}^{\prime} \boldsymbol{\Sigma}^{-1} \mathbf{e}_{i} \lambda_{i}}{\alpha_{i}} \cosh \left(2 \lambda_{i} \mathbf{e}_{i}^{\prime} \boldsymbol{\Sigma}^{-1}(\mathbf{y}-\gamma)\right)\right] \\
h\left\{\sum_{i=1}^{n} \frac{4}{\alpha_{i}^{2}} \sinh ^{2}\left(2 \lambda_{i} \mathbf{e}_{i}^{\prime} \boldsymbol{\Sigma}^{-1}(\mathbf{y}-\boldsymbol{\gamma})\right)\right\},
\end{aligned}
$$

where $\mathbf{y} \in \Re^{n}$, and $\mathbf{e}_{i}$ is the $i$-th vector of the canonical base in $\Re^{n}$. Here we write this fact by $\mathbf{Y} \sim \mathcal{G S E}_{n}^{I}(\boldsymbol{\alpha}, \boldsymbol{\gamma}, \boldsymbol{\Sigma}, \boldsymbol{\lambda} ; h)$.

Once again we note that if $\lambda_{i}=0.5$, for every $i=1, \ldots, n$, it is obtained the multivariate sinh-spherical distribution type I, see Theorem 5.2.

\section{Some extensions}

Let $\mathbf{A} \in \Re^{m \times m}$ be a positive definite matrix with spectral decomposition $\mathbf{A}=\mathbf{H D H}^{\prime}$, where $\mathbf{H}$ is an orthogonal matrix, and $\mathbf{D}=\operatorname{diag}\left(\lambda_{1}, \ldots, \lambda_{m}\right)$ is a diagonal matrix such that $\lambda_{1}>\cdots>\lambda_{m}>0$; if $f(x)$ is a differentiable function of $x$ and $\mathbf{F}(\mathbf{D})=\operatorname{diag}\left(f\left(\lambda_{1}\right), \ldots, f\left(\lambda_{m}\right)\right)$; then we define $\mathbf{F}(\mathbf{A})=\mathbf{H F}(\mathbf{D}) \mathbf{H}^{\prime}$. For example, if $f(x)=\sqrt{x}$, then $\mathbf{F}(\mathbf{A})=\mathbf{A}^{1 / 2}$ defines the non-negative definite square root of A. Similarly, if $f(x)=x^{-1}$, then $\mathbf{F}(\mathbf{A})=\mathbf{A}^{-1}$ defines the inverse of the matrix A, see [24, p. 39], [17, pp. 95-96] and [9]. In function of the spectral decomposition and the singular value decomposition, SVD, this result was generalised by [9] to semi-definite positive matrices and general rectangular matrices, respectively. With these generalisations we can define the function of a random matrix in an alternative way: instead of the definition of the logarithm of a matrix $\mathbf{A}=\left(a_{i j}\right)$ in the classical way $\ln (\mathbf{A})=\left(\ln \left(a_{i j}\right)\right)$, we can define it like $\ln (\mathbf{A})=\mathbf{H} \ln (\mathbf{D}) \mathbf{H}^{\prime}$. In particular, these results are applicable to the case of a random vector. But, if $\mathbf{X} \in \Re^{m}$, the SVD of the vector $\mathbf{X}$ is given simply by $\mathbf{X}=\rho \mathbf{W}$, where $\mathbf{W} \in \Re^{m}$ is $\|\mathbf{W}\|^{2}=1$ and $\rho=\|\mathbf{X}\|$, and here the vectorial cases for the SVD, the QR decomposition and the polar decomposition coincide, see [7]. Moreover, the decomposition $\mathbf{X}=\rho \mathbf{W}$, for a random vector with spherical (or elliptical) distribution, is known in the literature as the stochastic representation of the vector $\mathbf{X}$, see [12, Section 2.5.1] or [11, Theorem 2.3, p. 30]. Also, it is known that $(d \mathbf{X})=\rho^{m-1} d \rho\left(\mathbf{W}^{\prime} d \mathbf{W}\right)$, see [7] or [?]; then we have the following result: 
Theorem 7.1 Let $\mathbf{X} \in \Re^{m}$ be a vector such that its $S V D$ is given by $\mathbf{X}=$ $\rho \mathbf{W}$, with $\rho>0$ y $\mathbf{W} \in \Re^{m}$ con $\|\mathbf{W}\|^{2}=1$. Let $f(x)$ be a differentiable function in $x$ and define $\mathbf{F}(\mathbf{X})=f(\rho) \mathbf{W}$. Then

$$
(d \mathbf{F}(\mathbf{X}))=\left(\frac{f(\rho)}{\rho}\right)^{m-1}\left(\frac{d f(\rho)}{d \rho}\right)(d \mathbf{X}),
$$

where, as before, $(d \mathbf{X})$ denotes the exterior product, see [?, pp. 52-53].

Proof: The demonstration is similar to that one given in [9] for the general case.

As consequences of the present section we can establish the following results:

Corollary 7.2 Let $\mathbf{U} \sim \mathcal{E}_{n}(\mathbf{0}, \mathbf{I} ; h)$ be such that $\mathbf{U}=\mathbf{F}(\mathbf{T})=f(\rho) \mathbf{W}$, where $\mathbf{T}=\rho \mathbf{W}$ is the $S V D$ of the random vector $\mathbf{T}$, with $\rho>0, \mathbf{W} \in \Re^{m}$ and $\|\mathbf{W}\|^{2}=1$; so $f(\rho)$ is given by

$$
f(\rho)=\frac{1}{\alpha}\left[\sqrt{\frac{\rho}{\beta}}-\sqrt{\frac{\beta}{\rho}}\right], \alpha>0, \beta>0 .
$$

The distribution of the vector $\mathbf{T}=\left(T_{1}, \ldots, T_{n}\right)^{\prime}$ shall be termed the multivariate generalised Birnbaum-Saunders distribution type II, with density function:

$$
g_{\mathbf{T}}(\mathbf{t})=\frac{c(\|\mathbf{t}\|-\beta)^{n-1}(\|\mathbf{t}\|+\beta)}{2(\alpha \sqrt{\beta})^{n}\|\mathbf{t}\|^{3 n / 2}} h\left\{\frac{(\|\mathbf{t}\|-\beta)^{2}}{\alpha^{2} \beta\|\mathbf{t}\|}\right\},
$$

which shall be denoted by $\mathbf{T} \sim \mathcal{G B S}_{n}^{I I}(\alpha \mathbf{1}, \beta \mathbf{1} ; h)$, with $\mathbf{1}: n \times 1=(1, \ldots, 1)^{\prime}$.

Similarly, for a generalised three parameter Birnbaum-Saunders distribution we have the following result:

Corollary 7.3 Suppose that $\mathbf{U} \sim \mathcal{E}_{n}(\mathbf{0}, \mathbf{I} ; h)$, and consider the transformation

$$
f(\epsilon)=\frac{1}{\alpha}\left[\left(\frac{\epsilon}{\beta}\right)^{\lambda}-\left(\frac{\beta}{\epsilon}\right)^{\lambda}\right], \lambda>0, \quad \beta>0, \quad \alpha>0,
$$

with $\mathbf{U}=\mathbf{F}(\mathbf{V})=f(\epsilon) \mathbf{W}$, where $\mathbf{V}=\epsilon \mathbf{W}$ is the $S V D$ of the random vector $\mathbf{V}$, for $\epsilon>0$ and $\mathbf{W} \in \Re^{m}$ with $\|\mathbf{W}\|^{2}=1$. The density of the vector $\mathbf{V}$ is

$$
\begin{array}{r}
g_{\mathbf{v}}(\mathbf{v})=\frac{c \lambda}{(\alpha\|\mathbf{v}\|)^{n}}\left[\left(\frac{\|\mathbf{v}\|}{\beta}\right)^{\lambda}-\left(\frac{\beta}{\|\mathbf{v}\|}\right)^{\lambda}\right]^{n-1}\left[\left(\frac{\|\mathbf{v}\|}{\beta}\right)^{\lambda}+\left(\frac{\beta}{\|\mathbf{v}\|}\right)^{\lambda}\right] \\
h\left\{\frac{1}{\alpha^{2}}\left[\left(\frac{\|\mathbf{v}\|}{\beta}\right)^{2 \lambda}+\left(\frac{\beta}{\|\mathbf{v}\|}\right)^{2 \lambda}-2\right]\right\},
\end{array}
$$

This shall be termed the multivariate generalised three parameter Birnbaum-

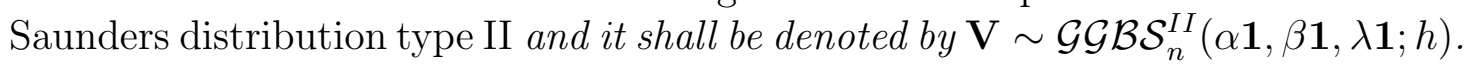


Next we give an extension of the multivariate sinh-spherical distribution:

Corollary 7.4 Suppose that $\mathbf{T} \sim \mathcal{G B S}_{n}^{I I}(\alpha \mathbf{1}, \beta \mathbf{1} ; h)$ and let $\mathbf{T}=\mathbf{F}(\mathbf{Y})=$ $f(\epsilon) \mathbf{P}$, with $f(\epsilon)=\exp \{2 \epsilon / \sigma\}$; where $\mathbf{Y}=\epsilon \mathbf{P}$ is the $S V D$ of $\mathbf{Y}$, for $\epsilon>0$, $\mathbf{P} \in \Re^{m}$ and $\|\mathbf{P}\|^{2}=1$. We say that $\mathbf{Y}$ has a multivariate sinh-spherical distribution type II of parameters $\alpha \mathbf{1}, \gamma \mathbf{1}$ with $\beta=\exp \{2 \gamma / \sigma\}$ and $\sigma \mathbf{I}$. Moreover, its density function is given by

$$
\begin{array}{r}
g_{\mathbf{Y}}(\mathbf{y})=\frac{2^{-n} c}{\sigma^{n} \alpha\|\mathbf{y}\|^{n-1}}\left(\cosh ^{2}\left(\frac{\|\mathbf{y}\|-\gamma}{\sigma}\right)-1\right)^{(n-1) / 2} \cosh \left(\frac{\|\mathbf{y}\|-\gamma}{\sigma}\right) \\
h\left\{\frac{4}{\alpha^{2}} \sinh ^{2}\left(\frac{\|\mathbf{y}\|-\gamma}{\sigma}\right)\right\},
\end{array}
$$

with $\mathbf{y} \in \Re^{n}$. We write this fact like $Y \sim \mathcal{S E}_{n}^{I I}(\alpha \mathbf{1}, \gamma \mathbf{1}, \sigma \mathbf{I} ; h)$.

Now, the result of Theorem 7.4 is extended to the generalised three parameter Birnbaum-Saunders distribution.

Corollary 7.5 If $\mathbf{V} \sim \mathcal{G G B}-\mathcal{S}_{n}^{I I}(\alpha \mathbf{1}, \beta \mathbf{1}, \lambda \mathbf{1} ; h)$ and $\mathbf{V}=\mathbf{F}(\mathbf{Y})=f(\epsilon) \mathbf{N}$, con $f(\epsilon)=\exp \{2 \epsilon / \sigma\}$; where $\mathbf{Y}=\epsilon \mathbf{N}$ is the $S V D$ of $\mathbf{Y} ; \epsilon>0$ and $\mathbf{V} \in \Re^{m}$ with $\|\mathbf{N}\|^{2}=1$; then we say $\mathbf{Y}$ has a multivariate generalised sinh-spherical distribution type II of parameters $\alpha \mathbf{1}, \gamma \mathbf{1}$ with $\beta=\exp \{2 \gamma / \sigma\}$ and $\sigma \mathbf{I}$. And its density function is given by

$$
\begin{aligned}
g_{\mathbf{Y}}(\mathbf{y})= & \frac{2^{1-n} c \lambda}{\sigma \alpha^{n}\|\mathbf{y}\|^{n-1}}\left\{\cosh ^{2}\left[2 \lambda\left(\frac{\|\mathbf{y}\|-\gamma}{\sigma}\right)\right]-1\right\}^{(n-1) / 2} \\
& \times \cosh \left[2 \lambda\left(\frac{\|\mathbf{y}\|-\gamma}{\sigma}\right)\right] h\left\{\frac{4}{\alpha^{2}} \sinh ^{2}\left[2 \lambda\left(\frac{\|\mathbf{y}\|-\gamma}{\sigma}\right)\right]\right\},
\end{aligned}
$$

with $\mathbf{y} \in \Re^{n}$. This fact shall be denoted by $Y \sim \mathcal{G S E}_{n}^{I I}(\alpha \mathbf{1}, \gamma \mathbf{1}, \sigma \mathbf{I} ; h)$.

An alternative expression for a log-elliptical distribution is the following:

Corollary 7.6 Suppose that $\mathbf{Y}=\ln (\mathbf{W}) \sim \mathcal{E} l_{n}(\boldsymbol{\mu}, \boldsymbol{\Sigma} ; h)$. Then we say $\mathbf{W}$ has a multivariate log-elliptical distribution type II and its density function is given by:

$$
g_{\mathbf{W}}(\mathbf{W})=\frac{c \ln ^{n-1}(\|\mathbf{w}\|)}{|\boldsymbol{\Sigma}|^{1 / 2}\|\mathbf{w}\|^{n}} h\left\{(\log (\mathbf{w})-\boldsymbol{\mu})^{\prime} \boldsymbol{\Sigma}^{-1}(\log (\mathbf{w})-\boldsymbol{\mu})\right\}, \quad \mathbf{w} \in \Re_{+}^{n}
$$

with $\log (\mathbf{w})=\ln (\kappa) \mathbf{M}$, and $\mathbf{W}=\kappa \mathbf{M}$ is the $S V D$ of the vector $\mathbf{W}$; where $\kappa>0, \mathbf{M} \in \Re^{m}$ and $\|\mathbf{M}\|^{2}=1$. This shall denote like $\mathbf{W} \sim \mathcal{L} \mathcal{E} l_{p}^{I I}(\boldsymbol{\mu}, \boldsymbol{\Sigma} ; h)$. 
Note that the results based on Lemma 7.1 (corollaries 11 - 14) can be generalised still more. For example, in Theorem 7.2, now suppose that $\mathbf{U} \sim$ $\mathcal{E}_{n}(\boldsymbol{\mu}, \boldsymbol{\Sigma} ; h), \boldsymbol{\mu} \in \Re^{n}$ and $\boldsymbol{\Sigma} \in \Re^{n \times n}$ with $\boldsymbol{\Sigma}>0$, then we obtain

$g_{\mathbf{T}}(\mathbf{t})=\frac{c\|\mathbf{t}\|^{-3 n / 2}(\|\mathbf{t}\|-\beta)^{n-1}(\|\mathbf{t}\|+\beta)}{2(\alpha \sqrt{\beta})^{n}|\mathbf{\Sigma}|^{1 / 2}} h\left\{(\mathbf{F}(\mathbf{t})-\boldsymbol{\mu})^{\prime} \boldsymbol{\Sigma}^{-1}(\mathbf{F}(\mathbf{t})-\boldsymbol{\mu})\right\}$

with $\mathbf{F}(\mathbf{t})=f(\rho) \mathbf{W}$, where $\mathbf{T}=\rho \mathbf{W}$ is the SVD of the random vector $\mathbf{T}$, for $\rho>0, \mathbf{W} \in \Re^{m}$ and $\|\mathbf{W}\|^{2}=1$. Here $f(\rho)$ is given by

$$
f(\rho)=\frac{1}{\alpha}\left[\sqrt{\frac{\rho}{\beta}}-\sqrt{\frac{\beta}{\rho}}\right], \alpha>0, \beta>0 .
$$

An interesting point to highlight comes from the fact that the families of distributions derived in Corollaries 11- 14 keep being families of spherical distributions in $\Re^{n}$ and $\Re_{+}^{n}$; then many of their properties: moments, marginals, conditionals, etc. can be obtained as particular cases of the general results derived for the families of spherical distributions, see [12] or [11]. Moreover, note that from those distributions could be generate the corresponding families of elliptical distributions just by defining $\mathbf{T}=\boldsymbol{\Sigma}^{-1 / 2}(\mathbf{X}-\boldsymbol{\mu})$, with $\boldsymbol{\Sigma}>\mathbf{0}$ and $\boldsymbol{\mu} \in \Re^{n}$. For example, the corresponding elliptical expression for the density (12) is given by

$$
\begin{array}{r}
g_{\mathbf{X}}(\mathbf{x})=\frac{c\left(\left[(\mathbf{x}-\boldsymbol{\mu})^{\prime} \boldsymbol{\Sigma}^{-1}(\mathbf{x}-\boldsymbol{\mu})\right]^{1 / 2}-\beta\right)^{n-1}\left(\left[(\mathbf{x}-\boldsymbol{\mu})^{\prime} \boldsymbol{\Sigma}^{-1}(\mathbf{x}-\boldsymbol{\mu})\right]^{1 / 2}+\beta\right)}{2(\alpha \sqrt{\beta})^{n}|\mathbf{\Sigma}|^{1 / 2}\left[(\mathbf{x}-\boldsymbol{\mu})^{\prime} \mathbf{\Sigma}^{-1}(\mathbf{x}-\boldsymbol{\mu})\right]^{3 n / 4}} \\
\times h\left\{\frac{\left(\left[(\mathbf{x}-\boldsymbol{\mu})^{\prime} \boldsymbol{\Sigma}^{-1}(\mathbf{x}-\boldsymbol{\mu})\right]^{1 / 2}-\beta\right)^{2}}{\alpha^{2} \beta\left[(\mathbf{x}-\boldsymbol{\mu})^{\prime} \boldsymbol{\Sigma}^{-1}(\mathbf{x}-\boldsymbol{\mu})\right]^{1 / 2}}\right\} .
\end{array}
$$

We finish this section noting that for a sample of dependent life data $X_{1}, \ldots, X_{n}$, the expressions of the densities given in Corollaries 11-15, can be consider with their respective likelihood functions, according to the case.

\section{Applications}

This sections starts showing some graphics of different densities like: the generalised three parameter Birnbaum-Saunders distribution (see Figure 1), the log-elliptical distribution (see Figure 2) and the sinh-spherical distribution (see Figure 3).

Next we explain, in an example, the way to estimate some of the distributions derived in the preceding sections: we took the first set of independent 
data given in [4] and we simulated a set of dependent data, for them the parameters are estimated and the corresponding criterion of information of Shcwarz is calculated, see [25].

\begin{tabular}{||c|c|c|c|c|c|c||}
\hline \hline Distribution & $S I C$ & LogVer & NP & $\mu$ & $s^{2}$ & $\nu$ \\
\hline \hline Special Case & 922.5638 & -456.6668 & 2 & 4.882796 & 0.038613230 & \\
Laplace & 923.0040 & -456.8869 & 2 & 4.890352 & 0.016497691 & \\
Normal & 923.4683 & -457.1190 & 2 & 4.881763 & 0.028737664 & \\
Pearson VII & 925.2058 & -455.6802 & 3 & 4.886503 & 0.182448431 & 4.676901 \\
T & 925.2058 & -455.6802 & 3 & 4.886503 & 0.021840419 & 8.353855 \\
Cauchy & 947.8048 & -469.2873 & 2 & 4.897028 & 0.008428758 & \\
\hline \hline
\end{tabular}

Table 1: Fit of the Log-Elliptical distributions for the independent case, ordered according to the $S I C$ criterion from lowest to highest. NP denotes the number of parameters considered in the optimisation.

\begin{tabular}{||c|c|c|c|c|c|c||}
\hline \hline Distribution & $S I C$ & LogVer & NP & $\mu$ & $s^{2}$ & $\nu$ \\
\hline \hline Normal & 867.20886 & -428.99926 & 2 & 5.3069933 & 0.007658998 & \\
Laplace & 867.90034 & -429.34500 & 2 & 5.3069934 & 0.000076589 & \\
Special Case & 870.26121 & -430.52544 & 2 & 5.3069933 & 0.132657078 & \\
Cauchy & 872.12753 & -431.45859 & 2 & 5.3069934 & 0.007658263 & \\
T & 872.77665 & -429.48057 & 3 & 5.3069949 & 0.007658495 & 62.115737 \\
Pearson VII & 873.99107 & -430.08778 & 3 & 5.3069934 & 0.100452931 & 56.557866 \\
\hline \hline
\end{tabular}

Table 2: Fit of the Log-Elliptical distributions for the dependent case, ordered according to the SIC criterion from lowest to highest. NP denotes the number of parameters considered in the optimisation.

\begin{tabular}{||c|c|c|c|c|c|c||}
\hline \hline Distribution & SIC & LogVer & NP & $\alpha$ & $\beta$ & $\nu$ \\
\hline \hline Special Case & 922.30800 & -456.53888 & 2 & 0.39571929 & 131.95014 & \\
Laplace & 922.49944 & -456.63460 & 2 & 0.26014664 & 133.00000 & \\
Normal & 924.75657 & -457.76316 & 2 & 0.34605813 & 131.67771 & \\
T & 925.21941 & -455.68702 & 3 & 0.29346658 & 132.49943 & 7.1484897 \\
Pearson VII & 925.21941 & -455.68702 & 3 & 0.78461501 & 132.49952 & 4.0741357 \\
Cauchy & 946.59686 & -468.68331 & 2 & 0.18426419 & 133.89997 & \\
\hline \hline
\end{tabular}

Table 3: Fit of the generalised three parameters Birnbaum-Saunders distributions for the independent case, ordered according to the SIC criterion from lowest to highest. NP denotes the number of parameters considered in the optimisation. 


\begin{tabular}{||c|c|c|c|c|c|c||}
\hline \hline Distribution & $S I C$ & LogVer & NP & $\alpha$ & $\beta$ & $\nu$ \\
\hline \hline Normal & 867.42236 & -429.10601 & 2 & 0.175886865 & 201.77284 & \\
Laplace & 868.11384 & -429.45175 & 2 & 0.017588691 & 201.77315 & \\
Special Case & 870.47471 & -430.63219 & 2 & 0.732003896 & 201.77314 & \\
Cauchy & 872.34103 & -431.56534 & 2 & 0.175881535 & 201.77315 & \\
T & 872.99746 & -429.59097 & 3 & 0.175886986 & 201.77314 & 61.390864 \\
Pearson VII & 874.25650 & -430.22049 & 3 & 0.619121931 & 201.77314 & 56.195175 \\
\hline \hline
\end{tabular}

Table 4: Fit of the generalised three parameters Birnbaum-Saunders distributions for the dependent case, ordered according to the SIC criterion from lowest to highest. NP denotes the number of parameters considered in the optimisation.

\begin{tabular}{||c|c|c|c|c|c|c||}
\hline \hline Distribution & $S I C$ & LogVer & NP & $\alpha$ & $\gamma$ & $\nu$ \\
\hline \hline Special Case & -383.10426 & 196.16725 & 2 & 0.080578881 & 1.5853847 & \\
Laplace & -382.73764 & 195.98394 & 2 & 0.052799946 & 1.5872637 & \\
Normal & -380.98375 & 195.10700 & 2 & 0.070163281 & 1.5848798 & \\
$T$ & -380.23087 & 197.03812 & 3 & 0.060009231 & 1.5862995 & 7.5079185 \\
Pearson VII & -380.23087 & 197.03812 & 3 & 0.164429417 & 1.5862995 & 4.2539823 \\
Cauchy & -358.54091 & 183.88558 & 2 & 0.037500503 & 1.5888055 & \\
\hline \hline
\end{tabular}

Table 5: Fit of the sinh-spherical distribution under generalised three parameters Birnbaum-Saunders distributions for the independent case, ordered according to the $S I C$ criterion from lowest to highest. NP denotes the number of parameters considered in the optimisation.

\begin{tabular}{||c|c|c|c|c|c|c||}
\hline \hline Distribution & $S I C$ & LogVer & NP & $\alpha$ & $\gamma$ & $\nu$ \\
\hline \hline Normal & -528.44805 & 268.82920 & 2 & 0.032911007 & 1.6688907 & \\
Laplace & -527.75657 & 268.48346 & 2 & 0.003291101 & 1.6688907 & \\
Special Case & -525.39570 & 267.30302 & 2 & 0.136968769 & 1.6688907 & \\
Cauchy & -523.52938 & 266.36986 & 2 & 0.032913891 & 1.6688907 & \\
T & -522.88956 & 268.35253 & 3 & 0.032911013 & 1.6688907 & 63.054749 \\
Pearson VII & -521.72838 & 267.77194 & 3 & 0.123381789 & 1.6688907 & 57.027312 \\
\hline \hline
\end{tabular}

Table 6: Fit of the sinh-spherical distribution under generalised three parameters Birnbaum-Saunders distributions for the dependent case, ordered according to the SIC criterion from lowest to highest. NP denotes the number of parameters considered in the optimisation.

ACKNOWLEDGEMENTS. This study was carried out with the assistance of CONACYT-45974-F. 


\section{References}

[1] R. Azencott, Simulated annealing, John Wiley \& Sons, New York, 1992.

[2] R. E. Barlow, and F. Proschan, Mathematical theory of reliability, SIAM (Classics in applied mathematics), 1965/1996.

[3] Z. W. Birnbaum, and S. C. Saunders, A new family of life distributions, J. App. Prob. 6 (1969a), 319-327.

[4] Z. W. Birnbaum, and S. C. Saunders, Estimation for a family of life distributions with applications to fatigue, J. App. Prob. 6 (1969b), 328347.

[5] J. A. Díaz-García, and J. R. Domínguez Molina, An independent or dependent sample of life data: Distributions and Estimation, Comunicación Técnica, No. I-05-02 (PE/CIMAT), Guanajuato, México (2005), http://www.cimat.mx/biblioteca/RepTec/index.html?m=2.

[6] J. A. Díaz-García, and G. González-Farías, A Note on the Cook's Distance, J. of Statist. Plan. and Inf. 120/1-2 (2004), 112-136.

[7] J. A. Díaz-García, and G. González-Farías, Singular Random Matrix Decompositions: Jacobians, J. of Multivariate Anal. 93/2 (2005a), 296-312.

[8] J. A. Díaz-García, and G. González-Farías, Singular Random Matrix Decompositions: Distributions, J. Multivariate Anal. 94/1 (2005b), 109-122.

[9] J. A. Díaz-García, and R. Gutiérrez-Jáimez, Functions of singular random matrices and its applications, TEST 14/2 (1997). In press.

[10] J. A. Díaz-García, and V. Leiva-Sánchez, A new family of life distributions based on elliptically contoured distributions, J. Statist. Plan. Inf. 128/2 (2005), 445-457.

[11] K. T. Fang, S. Kotz, and K. W. Ng, Symmetric multivariate and related distributions, Chapman and Hall, London, 1990.

[12] K. T. Fang, and Y. T. Zhang, Generalized multivariate analysis, SpringerVerlag, London, 1990.

[13] A. K. Gupta, and T. Varga, Elliptically Contoured Models in Statistics, Kluwer Academic Publishers, Dordrecht, 1993.

[14] J. D. Kalbfleish, and R. L. Prentice, The statistical analysis of failure time data, John Wilwy \& Sons, New York, 1980. 
[15] S. Kirkpatrick, C. D. Gelatt, and M. P. Vecchi, Optimization by simulated annealing, Science 220 (1983), 598-680.

[16] R. D. Leitch, Reliability analysis for engineers. An introduction, Oxford University Press Inc, 1995.

[17] A. M. Mathai, Jacobians of Matrix Transformations and Functions of Matrix Argument, World Scientific, New Jersey, 1997.

[18] mh:82 R. J. Muirhead, Aspects of multivariate statistical theory, Wiley Series in Probability and Mathematical Statistics, John Wiley \& Sons, Inc., New York, 1982.

[19] W. B. Nelson, and G. J. Hann, Linear estimation of a regression relationships from censured data, Part I - Simple methods and their applications (with discussion), Technometrics 14 (1972), 247-276.

[20] W. J. Owen, Another look at the Birnbaum-Saunders distribution, 2004, \http://www.stat.lanl.gov/MMR2004/Extended\%20Abstract/WOwnn.pdf.

[21] W. J. Owen, and W. J. Padgett, Accelerated test models for system strength based on Birnbaum-Saunders distribution, Lifetime Data Anal. 5 (1999), 133-147.

[22] J. R. Rieck, and J. R. Nedelman, A log-linear model for the BirnbaumSaunders distribution, Technometrics 33/1 (1991), 51-60.

[23] P. Siarry, G. Berthiau, F. Durbin, and J. Haussy, Enhanced simulated annealing for globally minimizing functions of many variables, ACM Trans. Math. Soft. 23/2 (1997), 209-228.

[24] M. S. Srivastava, and C. G. Khatri, An Introduction to Multivariate Statistics, North Holland, New York, 1979.

[25] G. Schwarz, Estimating the dimension of a model. Ann. Statist. 6 (1978), 461-464.

[26] P. Tobias, Reliability, Chapter 8 in e-Handbook of Statistical Methods, - Statistical Methods Group, NIST/SEMATECH, 2004, http://www.itl.nist.gov/div898/handbook/.

\section{Received: April 24, 2006}


Generalised Three Parameter Birnbaum-Saunders Distributions

(a) Bessel

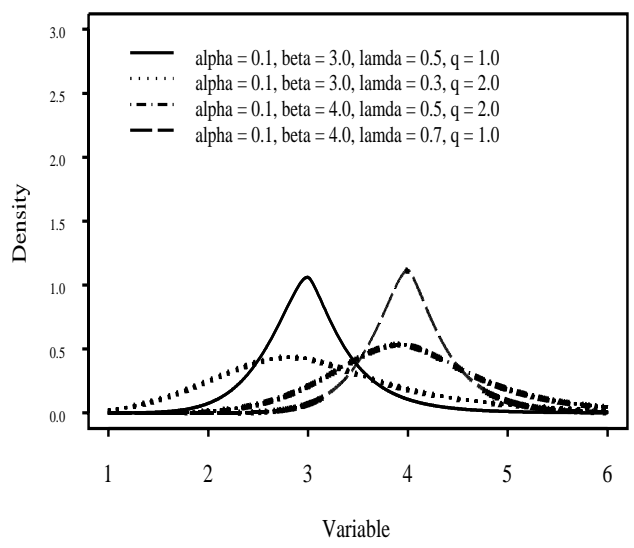

(c) Normal

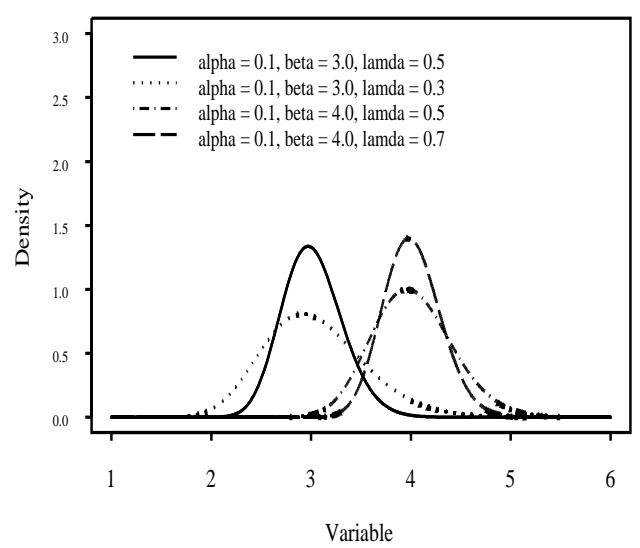

(b) Laplace

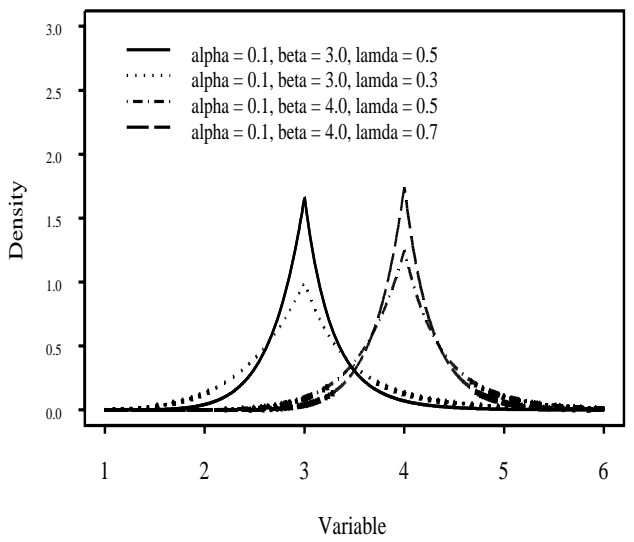

(d) Pearson VII

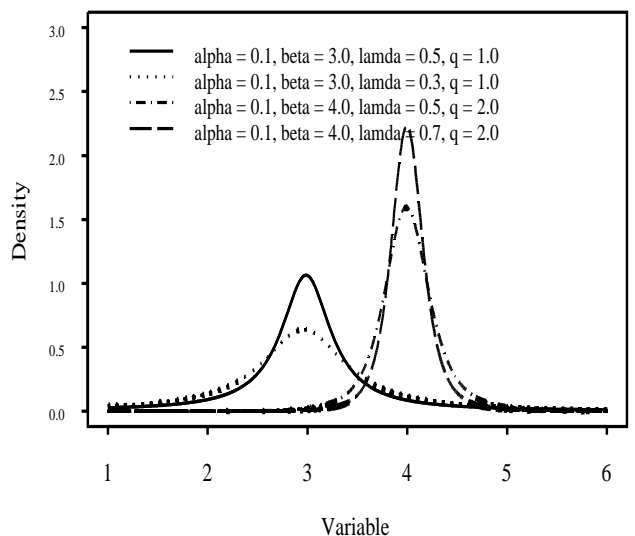

Figure 1: Densities of generalised three parameter Birnbaum-Saunders distribution type I 
(a) Bessel Log-Elliptical Distributions $\quad$ (b) Laplace

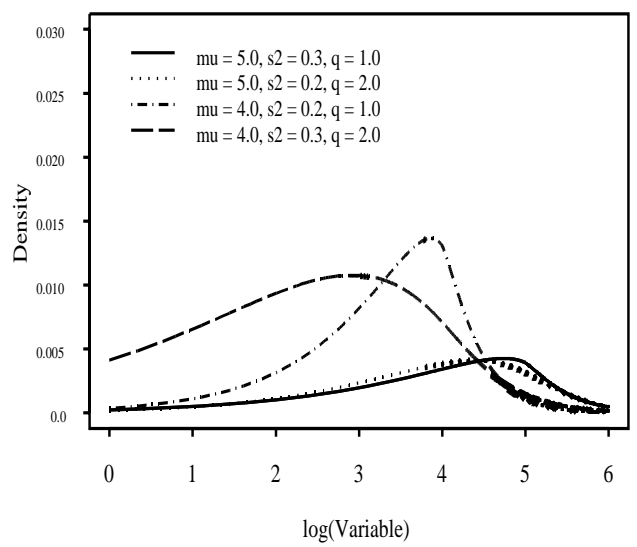

(c) Normal

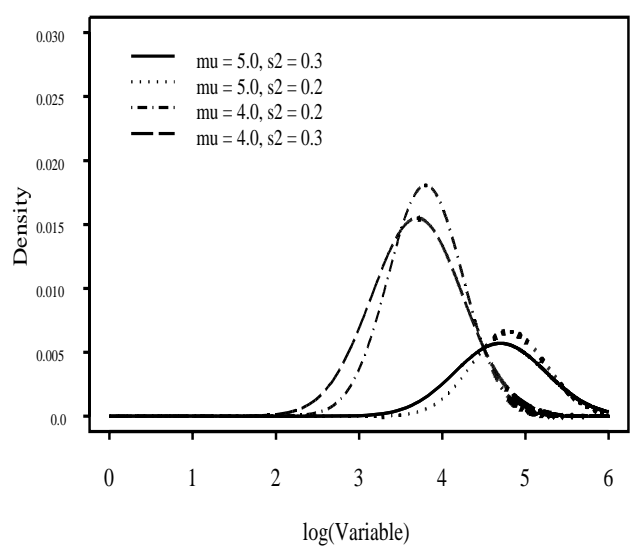

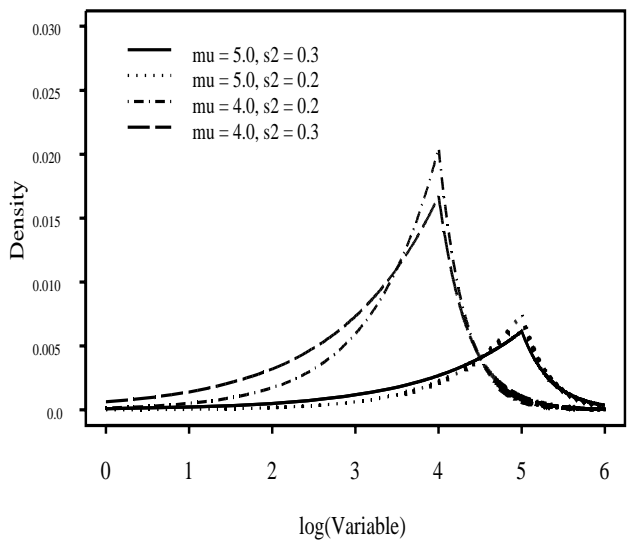

(d) PearsonVII

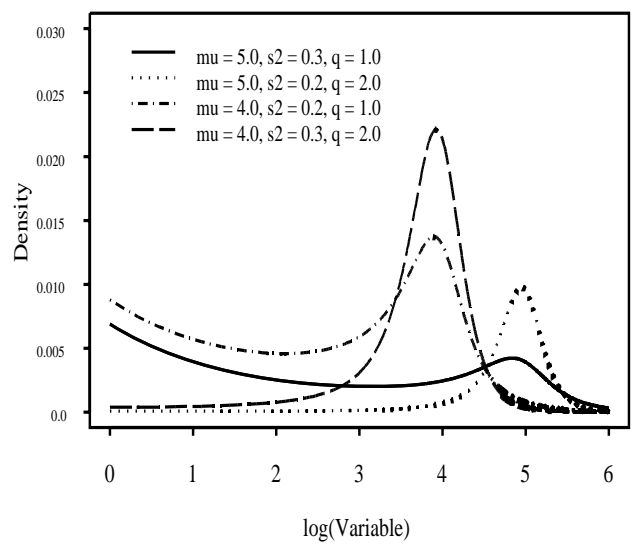

Figure 2: Densities of generalised log-elliptical distribution type I 
Sinh-Spherical Distributions Under Three Parameter Birnbaum-Saunders Distributions

(a) Bessel

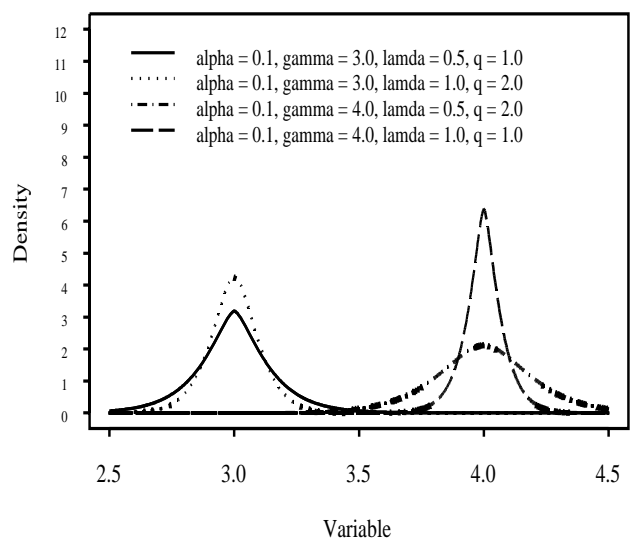

(c) Normal

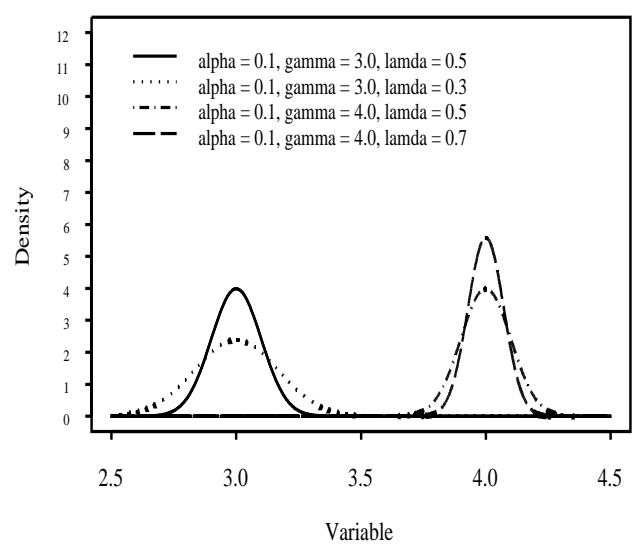

(b) Laplace

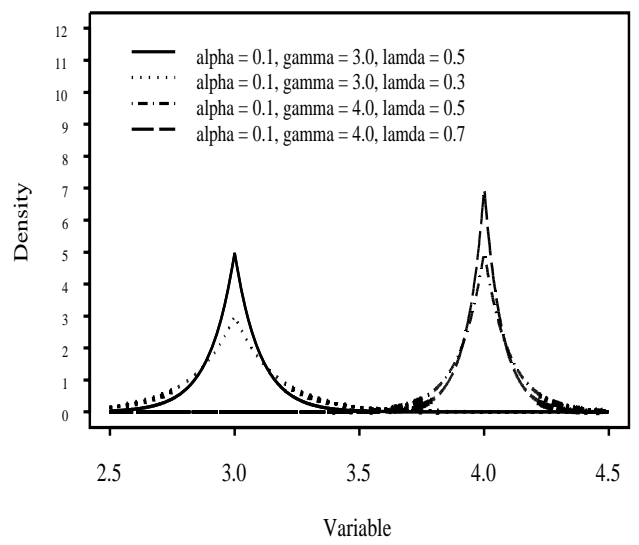

(d) PearsonVII

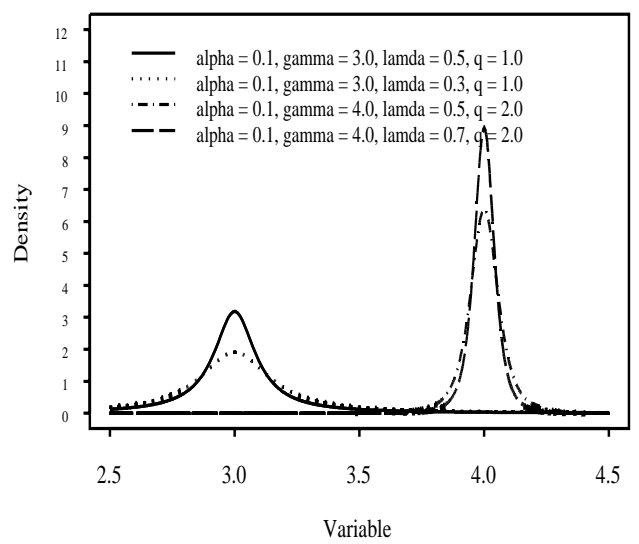

Figure 3: Densities of generalised sinh-spherical distribution type I 